\title{
The Formation and Characterization of Large Twin Related Domains
}

David B. Bober ${ }^{\mathrm{a}, \mathrm{b}}$, Jonathan Lind ${ }^{\mathrm{b}}$, Rupalee P. Mulay ${ }^{\mathrm{b}}$, Timothy J. Rupert ${ }^{\mathrm{a}, \mathrm{c}}$ and Mukul Kumar ${ }^{\mathrm{b} *}$

${ }^{a}$ Department of Mechanical and Aerospace Engineering, University of California, Irvine, CA 92697, USA

${ }^{\mathrm{b}}$ Lawrence Livermore National Laboratory, Livermore, CA 94550, United States

${ }^{c}$ Department of Chemical Engineering and Materials Science, University of California, Irvine, CA 92697, USA

*E-mail: kumar3@1lnl.gov

\section{Abstract}

The enhanced properties of grain boundary engineered metals are a result of their unique microstructures, which contain large clusters of twinned grains, called twin related domains. These large twin related domains in grain boundary engineered $\mathrm{Ni}$ were found to form through recrystallization. Orientation mapping showed that sparse nucleation and multiple twinning resulted in twin related domains containing hundreds of grains connected together in complex morphologies. A correlation was found between the size of the twin related domains and the overall twin boundary fraction. The same correlation was also observed in $\mathrm{Cu}$ and a $\mathrm{Ni}$ superalloy, showing that this is a general observation for grain boundary engineered microstructures. This finding can be understood through the topology of the twin related domains and an accompanying scaling relation is provided. The crystal orientations contained within each twin related domain were observed to depend on both the spatial correlation of twinning variants and the degree of branching in the twin boundary network. The results suggest a natural way of quantifying grain boundary engineered microstructures and provide a step toward making a closer connection between processing, microstructure, and performance.

\section{Keywords: Grain boundary engineering, twin related domain, recrystallization, microstructural evolution}




\section{Introduction}

Material properties can often be improved by controlling both the type and arrangement of grain boundaries, a process called grain boundary (GB) engineering [1]. These improvements have generally been linked to a profusion of twin boundaries, labeled as $\Sigma 3$ in the coincident site lattice (CSL) framework, making twin boundary fraction an important metric [2]. For example, Lin et al. [3] saw that the intergranular corrosion resistance of Inconel 600 was proportional to twin fraction. On the other hand, it has also been recognized that grain boundary topology, which cannot be assessed from boundary fraction, affects crack propagation $[4,5]$. Taking a more holistic view, Gertsman and Henager [6] observed that clusters of many grains were mutually connected by twin boundaries in $\mathrm{GB}$ engineered $\mathrm{Cu}-\mathrm{Ni}$. Within each cluster, every grain was related by a $\Sigma 3^{\mathrm{n}}$ misorientation [6]. This clustering concept was formalized by Reed and Kumar, who called such features twin related domains (TRDs) and offered a mathematical framework for their analysis [7]. Reed et al. [8] then showed how the length scale of TRDs can be used explain the fracture roughness of GB engineered and conventionally processed $\mathrm{Ni}$ subjected to intergranular stress corrosion. Likewise, Lind et al. [9] showed that TRD size affects the progress of thermal grain coarsening. As a whole, these studies demonstrate how the concept of TRDs can be a valuable tool for understanding how GB engineering affects material properties.

With mounting evidence that TRDs are important microstructural features, it is desirable to know more about how they form. The statistical increase in $\Sigma 3^{\mathrm{n}}$ GBs brought about by lowstrain recrystallization has been known for some time [10]. The mechanism for this increase is $\Sigma 3$ twinning, with higher order $\Sigma 3^{\mathrm{n}}$ GBs being formed by incidental $\Sigma 3$ interactions [11]. Indeed, twinning is fundamental to recrystallization, providing the only mechanism for producing 
orientations significantly different than those present in the deformed material, as reviewed by Berger et al. [12]. Dynamic recrystallization experiments on single crystal copper showed that all of the new orientations form as the result of twin chains [13]. A similar result was found for recrystallized aluminum, despite its high stacking fault energy [14]. Twinning can start in the early stages of nucleation [15], and continue to occur during boundary migration [16]. The first of these twins tend to occur along the primary or conjugate slip planes of the deformed material [17], while subsequent twins are more likely to form parallel to the moving growth front [16]. Twinning is suppressed when the growth front becomes a $30-40^{\circ}<111>$ type boundary $[14,17]$. The areal density of these twins is proportional to the prior strain and inversely to the prior grain size [18].

While well understood on an individual basis, there remains more to learn about how these twinning events produce the sorts of large twin related domains that appear to be so important to the properties of GB engineered materials. Qualitatively, the thousand-member TRDs in a GB engineered material bear little resemblance to those in a conventionally processed one. Lind et al. [9] have shown that these materials have topological differences that must be considered in order to understand how large TRDs can be built from relatively few twins. It remains to employ this idea in a predictive relationship between TRD size and twin fraction. There also remains much to learn about the internal structure of GB engineered TRDs, and how it develops. Using metrics proposed by Cayron [19], Lind et al. inferred that TRDs in GB engineered materials contain fewer unique orientations relative to their size than do TRDs in conventionally processed materials. How this difference arises in two materials of identical stacking fault energy is currently unknown. 
In this paper, we seek to understand how the size, topology and internal structure of TRDs develop during recrystallization. TRD formation is investigated by a series of interrupted annealing experiments, combined with orientation mapping. TRD boundary topology is considered, along with the probability of TRD coalescence. This leads to a scaling relationship linking TRD size to the twin fraction of the microstructure at large. Finally, we focus on understanding the internal structure of TRDs by applying metrics previously proposed in the literature and by examining their graph structure. These results are then used to examine how large GB engineered TRDs compare to smaller conventional ones. Taken as a whole, this provides a description of how GB engineered microstructures form and how to naturally quantify them.

\section{Materials and Methods}

Varying degrees of cold work were applied to samples of commercial purity Ni (UNS N02201), oxygen-free electronic Cu (UNS C10100) and Inconel 718 (UNS N07718), followed by heat treatment. These materials were chosen because they can be readily grain boundary engineered and have a range of low to medium stacking fault energies. Inconel in particular is also of industrial significance in applications that can potentially benefit from GB engineering. The levels of deformation were selected to cover a range of GB engineering and conventional processes, with details presented in Table 1. The GB engineering treatments involve small deformations and are expected to produce high twin fractions, while the conventional processes have larger deformations and produce microstructures typical of commercially wrought material. Rolling was performed in a 10 inch mill, with no single-pass of less than 5\%, and heat treatments 
were performed in air. Sample thicknesses are also listed in Table 1 because larger samples were observed to require longer times for complete recrystallization, presumably because they reached temperature more slowly. Specimens were water quenched, except for the Inconel samples, which were air cooled. Different material lots are identified in Table 1 because initial grain size and trace impurity content could have an effect.

Standard metallography techniques were used to prepare samples for electron backscatter diffraction (EBSD). Final polishing was performed with electropolishing when the stored plastic strain was of interest, in other cases mechanical polishing with colloidal silica was considered sufficient. EBSD data was collected using a Quanta 200 scanning electron microscope (FEI, Hillsboro, OR) equipped with a Hikari XP2 EBSD camera (EDAX, Mahwah, NJ). The map area and step sizes were selected to be suitable for the grain and TRD sizes of each sample, as listed in Table 1. The grain sizes are given as the circle equivalent diameter of the mean grain area. The orientation data analysis began with a standard dilation cleanup using commercial software (EDAX). Except when considering stored plastic strain, each grain was assigned a single average orientation and a $5^{\circ}$ threshold was used for grain reconstruction.

Each TRD was reconstructed with a depth-first search for grains connected by $\Sigma 3$ boundaries. This algorithm builds the twin network by starting at a random grain and exploring along a branch of twinned grains until no new twins are found, at which point it backtracks to another unexplored branch and the process repeats until every branch has been traversed. This search routine was repeated until every grain in the microstructure had been assigned to its parent TRD. A restrictive $\pm 1^{\circ}$ threshold was applied to identify $\Sigma 3$ boundaries, in order to limit the influence of GBs that coincidentally have near- $\Sigma 3$ misorientations $[9,19]$. No higher order $\Sigma 3^{\mathrm{n}}$ boundaries were used to build TRDs because it has been shown that they do little to change 
the size of TRDs [9]. TRD size was characterized by the number of grains they contain rather than a physical length-scale, a method that allows for convenient comparison of TRDs across materials with different grain sizes. Within each TRD, the misorientation axis of every $\Sigma 3$ boundary was tracked, after the method of Reed and Kumar [7]. Symmetry operators were applied such that the four $\Sigma 3$ variants were always expressed relative to the arbitrary starting grain, since each variant corresponds to one of the four unique $<111>$ misorientation axes. These are then used to determine all of the $\Sigma 3^{\mathrm{n}}$ relationships within a TRD [7, 19]. This approach is necessary because the direct misorientation-based categorization of high order $\Sigma 3^{\mathrm{n}}$ relationships is impractical [19]. The number of $\Sigma 3^{\mathrm{n}}$ relationships increases exponentially with $\mathrm{n}$, and they start to cluster very closely in orientation space at large values of $\mathrm{n}$ [17]. Once identified, each TRD (Figure 1(a)) can be conveniently represented by a network graph (Figure 1(b)), where each node (dot) represents a grain and each edge (line) a twin boundary. Mathematically, this information was represented by an adjacency matrix $\left(T_{i j}\right)$, where each element records the twin variant connecting the $\mathrm{i}^{\text {th }}$ and $\mathrm{j}^{\text {th }}$ grains, also shown in Figure $1(\mathrm{c})$. Another matrix can be easily calculated to find the $\Sigma 3^{\mathrm{n}}$ relationships between every pair of grains in the TRD, following Reed's concatenation rules [7].

\section{Results and Discussion}

\section{Observations of TRD Formation during Annealing}

The formation of large TRDs was investigated by interrupting the annealing step of a GB engineering process. Sections of Ni were rolled to $5 \%$ and then annealed for times ranging from 5 to $10 \mathrm{~min}$. The microstructure after annealing for $5 \mathrm{~min}$ showed no change from the as-rolled 
state, with the grains remaining equiaxed and $16 \mu \mathrm{m}$ in diameter. After $7 \mathrm{~min}$, a few larger grains appeared in the microstructure, which are visible in the inverse pole figure (IPF) map in Figure 2(a). In this type of map, each pixel is assigned a color based on its orientation, which provides a means to visualize grains and texture. The subtle gradients in color within individual grains indicate lattice curvature, which is due to geometrically necessary dislocations (GNDs) accumulated during deformation. These color gradients can be seen more clearly in the small grains in Figure 3(a), which is enlarged from Figure 2(b) $(7.5 \mathrm{~min})$. The relative density of these defects can be approximated by analyzing the grain orientation spread (GOS) [20], also shown in Figure 2. GOS measures the average misorientation between every pixel in a grain and the grain's mean orientation [20]. It emphasizes grain-scale differences in GND density and residual plastic strain, although strictly speaking GOS lacks a quantitative relationship to either of these. Yellow/green grains in Figure 2 and Figure 3(b) have a high GOS, while blue grains are low GOS. After annealing for $7 \mathrm{~min}$, most of the grains showed the same level of GOS that was found prior to annealing. This indicates that the residual plastic strain imparted by rolling has not yet been removed by either recovery or recrystallization. The key exceptions are the small scattered blue areas. These are relatively strain-free and correspond to the large grains visible in the IPF map. The appearance of large, strain-free grains was more pronounced after $7.5 \mathrm{~min}$, as shown in Figure 2(b). After 8.5 min (Figure 2(c)), nearly the entire microstructure is composed of such grains and after $10 \mathrm{~min}$ (not shown), the change was complete.

The results presented in Figure 2 are a textbook example of primary recrystallization: the replacement of a deformed microstructure by new strain-free grains. Comparing the three GB maps in Figure 2, it is obvious that recrystallization increased the fraction of twin boundaries (red lines). Figure 4 plots the overall twin fraction for intermediate stages and confirms that it 
increases in concert with the recrystallized fraction. In contrast, the twin fractions within the recrystallized and deformed regions remain constant during the process. This indicates that the overall change in twin fraction occurs because the recrystallized microstructure replaces the original one, which is similar to the findings of Rohrer et al. [21]. Visually, this is apparent in Figure 2 where the recrystallized islands, blue in the GOS map, correspond with the clusters of twins in the GB map. Less obvious is that these recrystallized islands are also single TRDs. That is to say, all of the grains in each low GOS island are mutually connected by twins. This can be seen in the fourth row of Figure 2, where each TRD has been assigned an arbitrary color. It is likewise apparent in Figure 3(c), where the grain boundary map has been superimposed on an enlarged TRD map. This confirms that the large TRDs formed as the result of multiple twinning during recrystallization.

\section{TRD Formation as a Recrystallization Process}

Regarding the formation of TRDs as a recrystallization process, it remains to discuss why they become so large in GB engineered materials. We will first examine if each recrystallized TRD originates from a single nucleation event, or if they somehow coalesce. Two independently nucleated TRDs can coalesce into a single TRD if there is a $\Sigma 3$ at their impingement, as required by the definition of a TRD [9]. Warrington and Boon [22] give the probability $\left(P_{\Sigma 3}\right)$ of forming a $\Sigma 3$ in a random polycrystalline aggregate, which is only $\sim 0.02 \%$ for a $\pm 1^{\circ}$ tolerance. If the orientations along a TRD boundary were independent, the probability $(P)$ of coalescence would then be:

$$
P=1-\left(1-P_{\Sigma 3}\right)^{m}
$$

or 


$$
P \approx m P_{\Sigma 3}, \text { for large } m
$$

where $m$ is the number of grain boundaries along the TRD boundary. For two TRDs that share a 100 GB-long boundary, the probability of coalescence would only be $\sim 2 \%$. Therefore, TRD coalescence is unlikely to factor predominantly into TRD formation, especially during the early stages of their growth. Even were coalescence to occur, the connectivity would be minimal and through a $\Sigma 3$ that would probably be far from coherent. A $\Sigma 3$ that formed by this sort of coincidence would likely be far from the ideal CSL relationship because the two orientations involved would be independently fixed by their parent TRDs. A similar analysis applies to the probability of coalescence at a low angle boundary. Of course, the assumption that each nucleated orientation is independent may not always hold true. In particular, if the deformed material already contains large TRDs, then there is a high probability that nearby nuclei will reside in the same TRD and therefore have a $\Sigma 3^{\mathrm{n}}$ relationship. This observation may be relevant to the development of TRDs through iterative GB engineering processes.

Where TRD coalescence is not prevalent, their size will be fixed by the nucleation density, with more nuclei leading to smaller TRDs. From the extensive literature on recrystallization, it is well known that the nucleation density will increase with increasing strain. This is born out in Table 1, which shows that TRD size appears to be controlled by the level of cold work, with heavier deformations leading to smaller TRDs. This is in agreement with other observations of Ni recrystallized after 5-50\% strain [23]. Initial grain size and the density of second phase particles would also be expected the influence TRD size via their well known effects on nucleation density [24]. Minimizing TRD nucleation density would be desirable because of the benefits of large TRDs $[8,9]$. The useful upper limit on TRD size will then be set by the minimum strain required for recrystallization and dense multiple twinning. 
In the absence of twinning, the observed low nucleation density would have produced a microstructure $\sim 10$ times coarser than observed. This causes the TRD maps in Figure 2 to bear a strong resemblance to abnormal grain growth. In fact, early work on GB engineering attributed clusters of grains connected by special boundaries to abnormal grain growth and not primary recrystallization [25]. The two can be distinguished on the basis of the inhomogeneity of residual plastic strain [24]. In the case of primary recrystallization, intermediate stages will show inhomogeneous dislocation density, with a transition from high to low overall density as annealing progresses. In abnormal grain growth (secondary recrystallization), the dislocation density will remain low and homogenous. Figures 2 and 3 demonstrate that recrystallization, not abnormal grain growth is occurring. This distinction between primary recrystallization and abnormal grain growth helps to explain why so many twins develop. In primary recrystallization, inhomogeneous dislocation density provides a driving pressure $\sim 100$ times greater than grain boundary energy does for secondary recrystallization [24]. The propensity for twinning has been shown to be proportional to the strength of this driving force $[18,26,27]$. Indeed, a material which gains twins during primary recrystallization may lose some of them during subsequent grain growth [26].

From the IPF and GOS maps in Figure 2, we are confident that this GB engineering routine is a recrystallization process mediated by multiple twinning. The small deformation causes a low density of nucleation sites, from which large TRDs form by multiple twinning during boundary migration. These twinning events are driven by the gradient in dislocation density between the deformed and recrystallized regions, which would not be present in normal grain growth or secondary recrystallization. This is precisely the multiple twinning to which Gertsman and Henegar [6] attributed their early observation of TRDs, and also matches Lim and 
Raj's [10] even earlier conclusions. The result is TRDs that grow to contain many twins and eventually impinge, forming a GB engineered microstructure.

\section{Topological Models for TRD Growth}

The recrystallization process described above leads to a distinctive GB topology. Consider the arrangement of boundaries in Figure 3(c), which shows two large recrystallized TRDs and the surrounding unrecrystallized material. Based on the previous section, it is clear that the two large TRDs are in the process of consuming the smaller grained regions. Note that the boundary between these two groups cannot, by definition, contain twins; nor can the boundary between the two large TRDs. However, within each TRD, there is a profusion of twinning, and also many $\Sigma 9$ and $\Sigma 27$ boundaries, shown in blue. This pattern has consequences for the final microstructure, including the overall twin fraction and the network connectivity. How this topology develops, and its effects, are considered in the rest of this section.

We will start with a simple geometric model based on the Eden cluster growth model (ECGM) [28]. This is a purely geometric model that has been used to study growth processes as diverse as bacterial colonies [28] and the crystallization of amorphous Al-Ge [29]. We use the ECGM here to model an artificial grain structure and test the effect of TRD nucleation density on the overall microstructure. Artificial 'TRDs' were built by first assigning random orientations to a number of seed locations within a 2D hexagonal lattice, which are analogous to nucleation sites. A random quaternion was used to create a uniform orientation distribution. The next step was to assign a $\Sigma 3$-related orientation to a neighboring location, creating a 'twin'. More 'twins' were added to the growing clusters until the simulation cell was filled (10,000 grains). This is 
analogous to the multiple twinning events that occur during TRD growth. The twin variant was selected at random, rather than incorporating any of the selection biases present in real materials $[16,17]$. No orientation was changed after its initial assignment. This reflects the observation that grains within recrystallized TRDs are very stable [9]. To be clear, the ECGM does not include physical models for nucleation, growth or twinning. It is a set of geometric rules based on the phenomenology common to many growth processes [28]. The goal of such a model is to explore the geometrically controlled aspects of a process.

The structures created by the ECGM contain clusters of grains connected by ' $\Sigma$ ' boundaries and surrounded by an envelope of 'non- $\Sigma 3$ ' boundaries, much like the real TRDs already discussed. This can be seen in the example ECGM output in Figure 5, where ' $\Sigma$ ' boundaries are shown in red, TRD boundaries in black, and all others in gray. Examining instances of the ECGM having different seed densities, a correlation between 'twin' number fraction and 'TRD' size emerges. As 'TRD' size increases, the 'twin' fraction initially rose rapidly before eventually plateauing. This trend is shown as the dashed gray line in Figure 6, and it can be compared directly to real materials characterized via EBSD. The TRD sizes and twin fractions calculated for every material in Table 1 are plotted in Figure 6. TRD size was taken to be the quadratic mean of the number of grains per TRD because this weights them based on the fraction of the total microstructure they form. Given that there are no fitting parameters, the data agrees surprisingly well with the ECGM. It is interesting that this simple geometric model captures much of the TRD size dependence on $\Sigma 3$ content. Based on the model's very limited scope, this trend must be a geometric consequence of nucleation density.

This agreement between the ECGM and experimental data stems from a topological similarity between the two due to the external boundaries of a TRD being composed entirely of 
non- $\Sigma 3$ boundaries. By the same tautology, all of the $\Sigma 3$ boundaries lie within TRDs. From these two facts, a 2D geometrical argument can be constructed to explain the ratio of $\Sigma 3$ to non$\Sigma 3$ GBs. As a TRD grows, an increasing fraction of its grains will lie in the interior area than at the perimeter. The usual scaling of area $\left(\propto l e n g t h^{2}\right)$ versus perimeter $\left(\propto l e n g t h^{1}\right)$ will then cause microstructures with larger TRDs to have higher twin fractions. This can be stated mathematically as follows:

$$
f_{\Sigma 3}=\frac{c^{\prime} B_{\text {int }}}{B_{\text {int }}+B_{\text {ext }}}
$$

where $f_{\Sigma 3}$ is the $\Sigma 3$ number fraction, $B_{\text {int }}$ is the number of boundaries inside TRDs, and $B_{\text {ext }}$ is the number of grain boundaries around TRD perimeters. The constant c' assumes a fixed distribution of $\Sigma 3^{\mathrm{n}}$ boundaries inside TRDs. If we simplistically assume TRDs to be large circles composed of smaller circular grains, then Equation 3 can approximated as:

$$
f_{\Sigma 3} \approx c \frac{N-\sqrt{N}}{N}
$$

where $N$ is the TRD size, and $c$ is a proportionality constant that incorporates $c$ ' and geometric constants. Figure 6 shows the results of fitting Equation 4 to the EBSD data, shown as the solid line. Again, the agreement with the data is quite good, with a coefficient of determination or $\mathrm{r}$ squared value of 0.96 .

The limiting cases of Equation 4 are informative. In the trivial case of a microstructure composed of TRDs all having only 1 member, it correctly predicts a twin fraction of 0 . In the case of infinitely large TRDs, it requires that the twin fraction approach $c$, which a least squares fit shows to be $\sim 0.5$. Equivalently, we could say that at a twin fraction of $c$ (apparently $\sim 0.5$ ), TRDs approach infinite size. Infinite TRD size prohibits a percolating path of weak (non- $\Sigma 3$ ) 
boundaries because such paths only exist at TRD boundaries. This can be compared to the results of random percolation models. Accounting for triple junction constraints, Schuh et al. [5] predicted that a twin number fraction of $0.275-0.335$ would prevent percolation of non- $\Sigma 3^{\mathrm{n}}$ boundaries. These values are much lower than the $0.5 \Sigma 3$ threshold fraction that Equation 4 predicts. In fact, all of the microstructures studied here contained weak percolating paths, despite several having twin number fractions that exceed Schuh's predicted threshold. Preventing non- $\Sigma 3 \mathrm{~GB}$ percolation in GB engineered materials is apparently more difficult than random percolation models predict. This is similar to the observation of Basinger et al. [30], who noted that the non-random arrangement of grain boundaries could promote percolating paths. In essence, the formation of large TRDs introduces twins in an ordered way that, compared to randomly distributed twins, is inefficient at breaking up weak GB paths.

Equation 4 also predicts that a high twin fraction will only occur for microstructures composed of large TRDs. As previously discussed, TRD size is inversely related to the level of pre-recrystallization deformation and therefore twin fraction should be as well. Indeed, prior literature generally shows just such a trend for Ni [23, 31-33]. With one exception, all of the single iteration processes studied by Guyot and Richards [32] show this trend. That exception is the case of Ni deformed to $2.5 \%$ and recrystallized at $900{ }^{\circ} \mathrm{C}$, which showed a much lower twin fraction than did their sample deformed to 5\% strain [32]. A probable explanation for this data point is that the low strain was insufficient to cause recrystallization. Shimada et al. [34] found a similar trend in 304 stainless steel, with a peak special boundary content occurring for a $5 \%$ rolling reduction. This explains why many GB engineering treatments use strains in the range of 5-20\% [33, 35-38], even though absolute twin density increases with higher strains [26]. This also points toward a limit of current GB engineering techniques, and explains their apparent 
inability to create the ideal 'twin limited' microstructure. Ideally, the pre-recrystallization strain would be large enough to drive TRD growth and frequent twinning, but simultaneously small enough to reduce nucleation density to nearly zero. These goals are competing, if not necessarily incompatible.

\section{Characterization of Internal TRD Structure}

The two large TRDs in Figure 3 contain grains of many different orientations. This is quite different from the lamellar TRDs found in columnar nanotwinned materials [39]. While columnar TRDs can also contain many twins, they all share a common twinning plane and are composed of only two distinct orientations [39]. This type of difference would not be evident from the size or deformation state of the TRDs, which are the only distinctions that we have so far drawn. The orientations of a TRD's constituent grains therefore provide another valuable source of information. The goal of this section is to analyze the orientations within TRDs to understand more about how large TRDs form and how they may differ from smaller ones. This will also address if the TRDs in GB engineered materials differ from those in conventional materials by some characteristic other than size.

Cayron [19] proposed to characterize the diversity of orientation within a TRD using a metric called polysynthetism. The definition of polysynthetism $\left(P_{s}\right)$ is given by:

$$
P_{S}=\frac{N}{N_{o}}
$$

where, $N_{o}$ is the number of unique orientations within a TRD, as determined by reconstructing the twin graph [19]. Here, $N$ remains the number of grains in a TRD. This can be interpreted as 
an inverse measure of orientation diversity, with high values indicating relatively few unique orientations, compared to the TRD size.

We measured the polysynthetism of each TRD in all of the samples listed in Table 1, with the results plotted in Figure 7(a). The mean value at each TRD size is shown. Here we have again comingled the data from samples 1-15 because an individual analysis added little insight, which is in line with the materials' similar stacking fault energies [19]. The general trend is for small TRDs to possess a lower polysynthetism than larger ones. In other words, large TRDs in the GB engineered materials contain fewer unique orientations relative to their size than do the small TRDs in the conventionally processed materials. There is a kind of diminishing returns in which a growing TRD gains new grain orientations at a rate inversely related its size.

To understand how microstructure affects polysynthetism, synthetic twin network graphs were simulated. The simplest of these is a linear chain model, where one grain is twinned to a second, which is twinned to a third, and so forth. The only two parameters in this model are the length of the chain and the order of twin variants. The order of the twinning variants requires particular attention. As has been described by Reed and Kumar [7], grains connected by two consecutive and identical twin variants have the same orientation $(\Sigma 1)$. Consecutive, but nonrepeated, twinning generates a $\Sigma 9$ relationship. We will quantify this as the probability of repeated twinning $P_{r}$, defined as:

$$
P_{r}=\frac{n_{\Sigma 1}}{n_{\Sigma 1}+n_{\Sigma 9}}
$$

where $n_{\Sigma 1}$ is the number of $\Sigma 1$ relationships between second nearest neighbors in a TRD's twin graph, and $n_{\Sigma 9}$ is the like measure of $\Sigma 9 \mathrm{~s}$. A similar measure has also been proposed by Lin [40], but limited to 3-member TRDs. Intuitively, a high $P_{r}$ would be expected to produce a high 
polysynthetism. For a $P_{r}=1$, an infinitely long twin chain will have an infinite polysynthetism [19]. Similarly, an infinite twin chain with $P_{r}=0$ would have a polysynthetism of 1 . If each of the four possible twin variants were always equally probable, then the expected value of $P_{r}$ would be 0.25 . Using this value, many twin chains were generated, their polysynthetisms computed, and the means at a range of TRD sizes plotted in Figure 7(a). These are shown as the dashed gray line labeled ' $P_{r}=0.25, B=1$.' Obviously, this model does not reflect the data well, predicting only about $1 / 2$ the polysynthetism actually observed in the large TRDs. The arbitrary use of $P_{r}=0.25$ is a likely suspect for the poor agreement, there being little basis for assuming the twinning variant is selected at random $[16,17]$. Measuring the actual $P_{r}$ in the data sets, it was found that the mean value for all TRDs is about 0.3 , which reinforces the idea that twin variant selection is biased $[16,17]$. As can be seen in Figure $8(a), P_{r}$ is only weakly correlated to TRD size, having a Pearson correlation coefficient of -0.23 . Repeating the simulations with this new value of $P_{r}=0.30$, produced the line shown on Figure 7(a) that is labeled $P_{r}=0.30, B=1$. While the predicted polysynthetism is greater than it was for $P_{r}=$ 0.25 , it still falls well short of the data. This implies that the model is missing some important aspect(s) of TRD structure.

One possible inadequacy is that real TRDs are not composed of linear chains, instead having complicated topologies like the examples in Figures 1,2 and 3. To quantify this, we have adapted Bertz's [41] analysis of branching hydrocarbon chains. Our modification is to normalize Bertz's [41] branching factor by its minimum possible value at each TRD size. This new branching factor $(B)$ is given by,

$$
B=\frac{1}{2(N-2)} \sum_{i}^{N} d_{i}\left(d_{i}-1\right)
$$


where, $d_{i}$ is the degree of each node $i$ in a TRD's twin graph and $\mathrm{N}$ is still the number of grains in the TRD. The value of $d_{i}$ is equivalent to the number of twin-neighbors that each grain has. This metric is always 1 for linear chains and increases for star topologies, with larger stars having greater branching factor values. This provides a way to categorize the branching in twin graph networks. The intuitive nature of branching factor is shown in Figure 8(b), where example TRD graphs are arranged according to their size and branching factor. The branching factors measured for each TRD in the EBSD data are shown in Figure 8(c). The trend is for a branching factor that is low for small TRDs and higher for large TRDs. Large TRDs $(\mathrm{N}>100)$, have a mean $B$ value of 5.2 with a standard deviation of 1.9. To apply this network characteristic to the analysis of polysynthetism, several thousand random networks were generated with varying degrees of branching. This was done by growing them outward from a starting node, and at each new node determining the degree by rounding a lognormally distributed random number. This distribution is convenient for always producing a positive number, and is otherwise a somewhat arbitrary choice. The random distribution creates nodes with varying degree, like those observed in real twin networks. To get a range of networks, the mean and variance of the distribution were varied. The mean probability of repeat twinning was held near the measured value of 0.30 . This library of simulated TRDs was then sorted by branching factor and TRD size, and the mean values of polysynthetism calculated. In the extreme case of $B=1$, this model simplifies to the linear chain model. The results are plotted in Figure 7(a), where it can be seen that higher branching factors increase polysynthetism. Importantly, the values of $B$ where the simulations match the data best are within the range of the measured $B$ values.

Before discussing the implications of $P_{r}$ and $B$, it is worth checking if this analysis holds for metrics other than polysynthetism. The same line of reasoning and model generation was 
repeated to analyze the length of the longest twin chain (LLC) [19]. Also proposed by Cayron [19], LLC is defined as the highest $\Sigma 3^{\mathrm{n}}$ relationship that exists in a TRD, irrespective of adjacency. It is relatively straight forward to calculate using the methods outlined by Cayron [19], or Reed and Kumar [7]. Figure 7(b) shows the measured values of LLC for the same set of materials discussed for polysynthetism. The trend lines in Figure 7(b) were generated using the same synthetic twin graphs as for polysynthetism. Again, the simulations match the data at branching factor values within the measured range. This suggests that $P_{r}$ and $B$ are describing polynthetism and LLC in a microstructurally meaningful way.

The preceding paragraphs aimed to show that the probability of repeated twinning $\left(P_{r}\right)$ and branching factor $(B)$ measure important aspects of TRD structure. If this is accepted, then it is noteworthy that $P_{r}$ was not correlated with TRD size. That indicates the GB engineering does not significantly change the tendency for adjacent twins to share a misorientation axis. This similarity presumably comes from their shared formation by multiple twinning, with the same twin variant selection rules acting in each case. On the other hand, there is a significant shift in $B$ between the GB engineered and conventional microstructures. The higher branching factors in the GB engineered materials confirm the intuitive sense that GB engineered TRDs are different than similar sized clusters of lamellar twins, the latter having a branching factor of 1 . This is probably a consequence of the dimensionality of their growth, GB engineered TRDs being 3D and lamellar ones being essentially 1D and having no opportunity to branch. Other forms of TRD growth may exist that produce characteristic differences in the probability of repeated twinning or branching factor. Annealed nanocrystalline foils can form TRDs by quasi-2D annealing twinning [42], and are therefore something of an intermediate case between GB engineered (3D) and lamellar TRDs (1D). It would also be interesting to examine twinning 
caused by grain rotation in nanocrystalline metals, as has been observed in MD simulations [43].

Perhaps these rotation induced twins can be distinguished from annealing twins with these new metrics, which is currently difficult to infer [44].

\section{Conclusions}

Twin related domains are unique building blocks of grain boundary engineered microstructures. Interrupted annealing experiments confirmed the expectation that large TRDs form by a recrystallization process that involves a low nucleation density and multiple twinning. An analysis of TRD topology demonstrated how TRD size and twin fraction are related. This same analysis also suggested that percolation models overestimate the long range connectivity of $\Sigma 3$ networks. It was also shown that the constituent orientations of a TRD depend on the twin network's probability of repeated twinning and branching factor. The branching factor indicated that GB engineered TRDs are quite different than simple lamellar structures, most likely because of how they grow. In contrast, the probability of repeated twinning was nearly uncorrelated with TRD size, indicating a similar twin chain formation process.

\section{Acknowledgements}

This work was partly performed under the auspices of the U. S. Department of Energy by Lawrence Livermore National Laboratory under Contract DE-AC52-07NA27344. D.B.B., J.L., R.P.M., and M.K. were supported by the U.S. Department of Energy (DOE), Office of Basic Energy Sciences, Division of Materials Science and Engineering under FWP\# SCW0939. 
D.B.B. also acknowledges the support of the Livermore Graduate Scholar Program at Lawrence Livermore National Laboratory. T.J.R. acknowledges support from the National Science Foundation through a CAREER Award No. DMR-1255305. 
Table 1: Processing, EBSD, and microstructural parameters

Table 1 lists the rolling reduction, and heat treatment temperature and time for each material. Iterated steps are noted by the number of iterations followed by an ' $x$ ' and the details of the repeated step. The supplier lots and thickness after rolling are also included. The EBSD step and map sizes are listed, and can be compared to the grain and TRD sizes. Twin number fraction is also listed for comparison with other literature.

\begin{tabular}{|c|c|c|c|c|c|c|c|c|}
\hline ID & Material & Processing Method & $\begin{array}{l}\text { Thickness } \\
\text { (in) }\end{array}$ & $\begin{array}{l}\text { EBSD } \\
\text { step } \\
\text { size } \\
(\mu \mathrm{m})\end{array}$ & $\begin{array}{c}\text { EBSD } \\
\text { map } \\
\text { area } \\
\left(\mathrm{mm}^{2}\right)\end{array}$ & $\begin{array}{l}\text { Grain } \\
\text { size } \\
(\mu \mathrm{m})\end{array}$ & $\begin{array}{l}\text { TRD size } \\
\text { (quadratic } \\
\text { mean) }\end{array}$ & $\begin{array}{c}\text { Twin } \\
\text { number } \\
\text { fraction }\end{array}$ \\
\hline 1 & $\mathrm{Cu}(1)^{*}$ & $60 \%, 500{ }^{\circ} \mathrm{C} / 30 \mathrm{~min}$ & N/A & 1 & 4 & 17 & 4.7 & 0.25 \\
\hline 2 & $\mathrm{Cu}(1)^{*}$ & $2 \mathrm{x}\left(20 \%, 500{ }^{\circ} \mathrm{C} / 30 \mathrm{~min}\right)$ & N/A & 1 & 4 & 32 & 18.4 & 0.36 \\
\hline 3 & $\mathrm{Cu}(2)$ & $60 \%^{* *}, 280^{\circ} \mathrm{C} / 195 \mathrm{~min}$ & 0.133 & 0.5 & 0.25 & 7 & 5.3 & 0.27 \\
\hline 4 & $\mathrm{Cu}(2)$ & $60 \%{ }^{* *}, 500{ }^{\circ} \mathrm{C} / 30 \mathrm{~min}$ & 0.133 & 0.5 & 1 & 8 & 2.4 & 0.17 \\
\hline 5 & $\mathrm{Cu}(2)$ & $\left(60 \%{ }^{* *}, 500{ }^{\circ} \mathrm{C} / 30 \mathrm{~min}\right)+\left(20 \%, 500{ }^{\circ} \mathrm{C} / 10 \mathrm{~min}\right)$ & 0.107 & 0.5 & 1 & 12 & 16.7 & 0.35 \\
\hline 6 & $\mathrm{Cu}(2)$ & $\left(60 \%^{* *}, 500{ }^{\circ} \mathrm{C} / 30 \mathrm{~min}\right)+2 \mathrm{x}\left(20 \%, 500{ }^{\circ} \mathrm{C} / 10 \mathrm{~min}\right)$ & 0.086 & 0.5 & 1 & 18 & 24.4 & 0.39 \\
\hline 7 & $\mathrm{Cu}(2)$ & $\left(60 \%^{* *}, 500{ }^{\circ} \mathrm{C} / 30 \mathrm{~min}\right)+3 \mathrm{x}\left(20 \%, 500{ }^{\circ} \mathrm{C} / 10 \mathrm{~min}\right)$ & 0.065 & 0.5 & 1 & 18 & 15.7 & 0.36 \\
\hline 8 & $\mathrm{Ni}(1)^{* * *}$ & $5 \mathrm{x}\left(20 \%, 900{ }^{\circ} \mathrm{C} / 15 \min \right)+900{ }^{\circ} \mathrm{C} / 60 \min$ & 0.413 & 2 & 4 & 54 & 3.0 & 0.22 \\
\hline 9 & $\mathrm{Ni}(1)^{* * *}$ & $3 \mathrm{x}\left(5 \%, 900{ }^{\circ} \mathrm{C} / 15 \mathrm{~min}\right)+400{ }^{\circ} \mathrm{C} / 24 \mathrm{~h}$ & 0.881 & 2 & 4 & 82 & 8.8 & 0.35 \\
\hline 10 & $\mathrm{Ni}(2)$ & $25 \%, 800{ }^{\circ} \mathrm{C} / 120 \mathrm{~min}$ & 0.286 & 2 & 4 & 96 & 1.9 & 0.14 \\
\hline 11 & $\mathrm{Ni}(2)$ & $5 \%, 900{ }^{\circ} \mathrm{C} / 15 \mathrm{~min}$ & 0.361 & 2 & 4 & 92 & 11.8 & 0.38 \\
\hline 12 & $\mathrm{Ni}(3)$ & $25 \%, 900{ }^{\circ} \mathrm{C} / 60 \mathrm{~min}$ & 0.388 & 2 & 4 & 62 & 2.0 & 0.12 \\
\hline 13 & $\mathrm{Ni}(3)$ & $5 \%, 900{ }^{\circ} \mathrm{C} / 15 \mathrm{~min}$ & 0.492 & 2 & 4 & 59 & 15.5 & 0.38 \\
\hline 14 & Inconel (1) & $25 \%, 1020^{\circ} \mathrm{C} / 30 \mathrm{~min}$ air cooled & 0.375 & 2 & 3 & 24 & 2.8 & 0.21 \\
\hline 15 & Inconel (1) & as-received, $1030^{\circ} \mathrm{C} / 60 \mathrm{~min}$ air cooled & 0.500 & 2 & 3 & 49 & 4.6 & 0.24 \\
\hline
\end{tabular}

*These materials are the same used in Blobaum et al. [38], and were produced with a forging operation.

** Rolled immediately after prolonged submersion in liquid nitrogen

***The materials are the same used in Bechtle et al. [33] 
Figure 1: (a) The inverse pole figure color map of an example TRD and its corresponding representations as (b) a network diagram and (c) an adjacency matrix. Red lines in part (a) are twin boundaries, with other GBs shown in black. Each point in (b) represents a grain in (a). Each line in (b) corresponds to a grain boundary in (a) and an entry in (c).

Figure 2: Orientation maps from a sample of Ni rolled 5\% and annealed at $900 \mathrm{C}$ for 7, 7.5, and $8.5 \mathrm{~min}$. The colors in the first row indicate grain orientation. In the second row, yellow/green colors indicate high residual deformation and blue indicates strain-free regions. The red lines in the third row are twin boundaries, black are TRD boundaries and all other GBs are gray. The colors in the fourth row identify each TRD.

Figure 3: (a) Enlarged IPF, (b) GOS, and (c) TRD maps from the 7.5 min sample shown in Figure 2. In (c), $\Sigma 3$ boundaries are represented by red lines, $\Sigma 9$ and $\Sigma 27$ by blue, TRD boundaries by black, and all others in gray.

Figure 4: Twin number fraction for intermediate annealing stages, showing consistently high twin fraction in the recrystallized area $($ GOS $<0.5)$, low twin fraction in the unrecrystallized area (GOS $>0.6)$, and an overall twin fraction that reflects the material's composite nature.

Figure 5: A close-up view of an Eden cluster growth model result. The red lines indicate 'twin' boundaries; black lines are 'TRD' boundaries. Other boundaries are shown in gray. The background color distinguishes each TRD.

Figure 6: Twin number fraction relative to twin related domain size are shown for the materials listed in Table 1. The predictions of the Eden cluster growth model and a new scaling relationship are shown as dashed and solid lines, respectively.

Figure 7: The measured values of polysynthetism (a) and the length of the longest twin chain (b) are plotted, with the mean value at each TRD size shown. Trend lines for simulated twin graphs with a range of repeated twinning probabilities $\left(P_{r}\right)$ and branching factors $(B)$ are also shown.

Figure 8: (a) The measured probability of repeated twinning, with the mean value at each TRD size shown. (b) Several example twin networks are plotted at positions corresponding to their sizes and branching factors and (c) the experimental values for branching factor. 


\section{References}

[1] Watanabe T. An approach to grain-boundary design for strong and ductile polycrystals, Res Mechanica 11 (1984) 47-84.

[2] Watanabe T. Grain boundary engineering: historical perspective and future prospects, Journal of Materials Science 46 (2011) 4095-4115.

[3] Lin P, Palumbo G, Erb U, Aust KT. Influence of grain boundary character distribution on sensitization and intergranular corrosion of alloy 600, Scr. Metall. Materialia 33 (1995) 1387-1392.

[4] Lim LC, Watanabe T. Fracture-Toughness And Brittle-Ductile Transition Controlled By GrainBoundary Character Distribution (GBCD) In Polycrystals, Acta Metallurgica Et Materialia 38 (1990) 25072516.

[5] Schuh CA, Minich RW, Kumar M. Connectivity and percolation in simulated grain-boundary networks, Philosophical Magazine 83 (2003) 711-726.

[6] Gertsman VY, Henager $\mathrm{CH}$. Grain boundary junctions in microstructure generated by multiple twinning, Interface Sci 11 (2003) 403-415.

[7] Reed BW, Kumar M. Mathematical methods for analyzing highly-twinned grain boundary networks, Scripta Materialia 54 (2006) 1029-1033.

[8] Reed BW, Kumar M, Minich RW, Rudd RE. Fracture roughness scaling and its correlation with grain boundary network structure, Acta Materialia 56 (2008) 3278-3289.

[9] Lind J, Li SF, Kumar M. Twin related domains in 3D microstructures of conventionally processed and grain boundary engineered materials, Acta Materialia 114 (2016) 43-53.

[10] Lim LC, Raj R. On the distribution of sigma for grain-boundaries in polycrystalline nickel prepared by strain-annealing technique, Acta Metallurgica 32 (1984) 1177-1181.

[11] Whitwham D, Mouflard M, Lacombe P, Ellis WC, Treuting RG. Atomic relationships in the cubic twinned state, J of Metals 3 (1951) 1070-1074.

[12] Berger A, Wilbrandt PJ, Ernst F, Klement U, Haasen P. On the generation of new orientations during recrystallization - recent results on the recrystallization of tensile-deformed fcc single-crystals, Prog Mater Sci 32 (1988) 1-95.

[13] Gottstein G, Zabardjadi D, Mecking H. Dynamic recrystallization in tension-deformed copper single crystals, Metal Science 13 (1979) 223-227.

[14] Berger A, Wilbrandt PJ, Haasen P. Development of the recrystallization texture in tensile deformed aluminum single-crystals, Acta Metallurgica 31 (1983) 1433-1443.

[15] Escher C, Gottstein G. Nucleation of recrystallization in boron doped Ni3Al, Acta Materialia 46 (1998) 525-539.

[16] Miura H, Sakai T, Mogawa R, Gottstein G. Nucleation of dynamic recrystallization at grain boundaries in copper bicrystals, Scripta Materialia 51 (2004) 671-675.

[17] Gottstein G. Annealing texture development by multiple twinning in fcc crystals, Acta Metallurgica 32 (1984) 1117-1138.

[18] Form W, Gindraux G, Mlyncar V. Density of annealing twins, Metal Science 14 (1980) 16-20.

[19] Cayron C. Quantification of multiple twinning in face centred cubic materials, Acta Materialia 59 (2011) 252-262.

[20] Wright SI, Nowell MM, Field DP. A review of strain analysis using electron backscatter diffraction, Microsc Microanal 17 (2011) 316-329.

[21] Rohrer GS, Bozzolo N, Jin Y, Lin B, Rollett AD, Bernacki M. Evolution of Microstructure in Pure Nickel during Processing for Grain Boundary Engineering. Mater. Sci. Forum, vol. 753, 2013. p.97-100.

[22] Warrington $\mathrm{DH}$, Boon M. Ordered structures in random grain-boundaries - some geometrical probabilities, Acta Metall. Mater. 23 (1975) 599-607. 
[23] Xia S, Zhou BX, Chen WJ. Grain cluster microstructure and grain boundary character distribution in alloy 690, Metall. Mater. Trans. A 40A (2009) 3016-3030.

[24] Rollett A, Humphreys FJ, Rohrer GS, Hatherly M. Recrystallization and related annealing phenomena, Elsevier Science, 2004.

[25] Randle V, Brown A. Development of grain misorientation texture, in terms of coincident site lattice structures, as a function of thermomechanical treatments, Philos. Mag. A-Phys. Condens. Matter Struct. Defect Mech. Prop. 59 (1989) 1075-1089.

[26] Jin Y, Lin B, Rollett AD, Rohrer GS, Bernacki M, Bozzolo N. Thermo-mechanical factors influencing annealing twin development in nickel during recrystallization, Journal of Materials Science 50 (2015) 5191-5203.

[27] Cahoon JR, Li Q, Richards NL. Microstructural and processing factors influencing the formation of annealing twins, Mater. Sci. Eng. A 526 (2009) 56-61.

[28] Eden M. A Two-dimensional Growth Process. Proc. Fourth Berkeley Symp. on Math. Stat. and Probab., vol. 4. Berkeley, Calif.: University of California Press, 1961. p.223-239.

[29] Lereah Y, Zarudi I, Grunbaum E, Deutscher G, Buldyrev SV, Stanley HE. Morphology of Ge-Al thin-films - experiments and model, Phys. Rev. E 49 (1994) 649-656.

[30] Basinger JA, Homer ER, Fullwood DT, Adams BL. Two-dimensional grain boundary percolation in alloy 304 stainless steel, Scripta Materialia 53 (2005) 959-963.

[31] Lee SL, Richards NL. The effect of single-step low strain and annealing of nickel on grain boundary character, Mater. Sci. Eng. A 390 (2005) 81-87.

[32] Guyot BM, Richards NL. A study on the effect of cold rolling and annealing on special grain boundary fractions in commercial-purity nickel, Mater. Sci. Eng. A 395 (2005) 87-97.

[33] Bechtle S, Kumar M, Somerday BP, Launey ME, Ritchie RO. Grain-boundary engineering markedly reduces susceptibility to intergranular hydrogen embrittlement in metallic materials, Acta Materialia 57 (2009) 4148-4157.

[34] Shimada M, Kokawa H, Wang ZJ, Sato YS, Karibe I. Optimization of grain boundary character distribution for intergranular corrosion resistant 304 stainless steel by twin-induced grain boundary engineering, Acta Materialia 50 (2002) 2331-2341.

[35] Thomson CB, Randle V. A study of twinning in nickel, Scripta Materialia 35 (1996) 385-390.

[36] Thaveeprungsriporn V, Was GS. The role of coincidence-site-lattice boundaries in creep of Ni16Cr-9Fe at 360 degrees C, Metall. Mater. Trans. A 28 (1997) 2101-2112.

[37] Kumar M, King WE, Schwartz AJ. Modifications to the microstructural topology in f.c.c. materials through thermomechanical processing, Acta Materialia 48 (2000) 2081-2091.

[38] Blobaum KJM, Stolken JS, Kumar M. Grain boundary engineering of copper shaped-charge. in: Schuh CA, Kumar M, Carter CB, Randle V, (Eds.). Interfacial Engineering for Optimized Properties lii, vol. 819. Materials Research Society, Warrendale, 2004. pp. 51-56.

[39] LaGrange T, Reed BW, Wall M, Mason J, Barbee T, Kumar M. Topological view of the thermal stability of nanotwinned copper, Appl. Phys. Lett. 102 (2013) 011905.

[40] Lin B. Investigating annealing twin formation mechanisms in face-centered cubic nickel. Carnegie Mellon University, 2015.

[41] Bertz SH. Branching in graphs and molecules, Discrete Applied Mathematics 19 (1988) 65-83.

[42] Simoes S, Calinas R, Vieira MT, Vieira MF, Ferreira PJ. In situ TEM study of grain growth in nanocrystalline copper thin films, Nanotechnology 21 (2010) 145701.

[43] Panzarino JF, Ramos JJ, Rupert TJ. Quantitative tracking of grain structure evolution in a nanocrystalline metal during cyclic loading, Modelling and Simulation in Materials Science and Engineering 23 (2015) 025005.

[44] Bober DB, Kumar M, Rupert TJ. Nanocrystalline grain boundary engineering: Increasing $\Sigma 3$ boundary fraction in pure Ni with thermomechanical treatments, Acta Mater. 86 (2015) 43-54. 


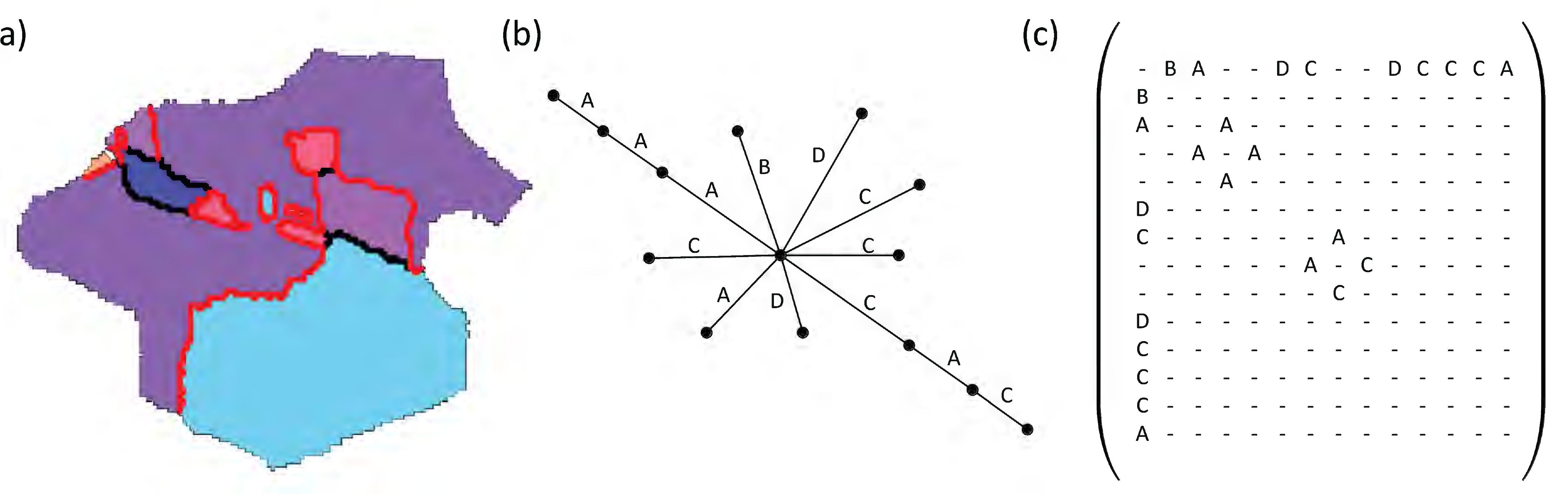

(b) (a)

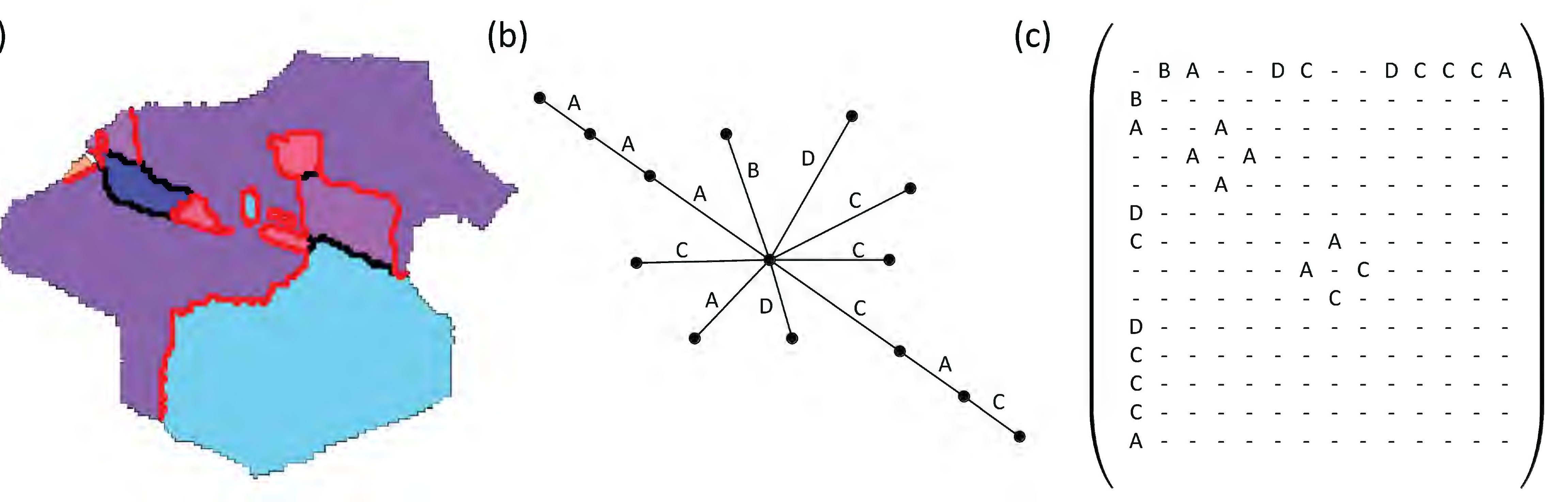

(c)
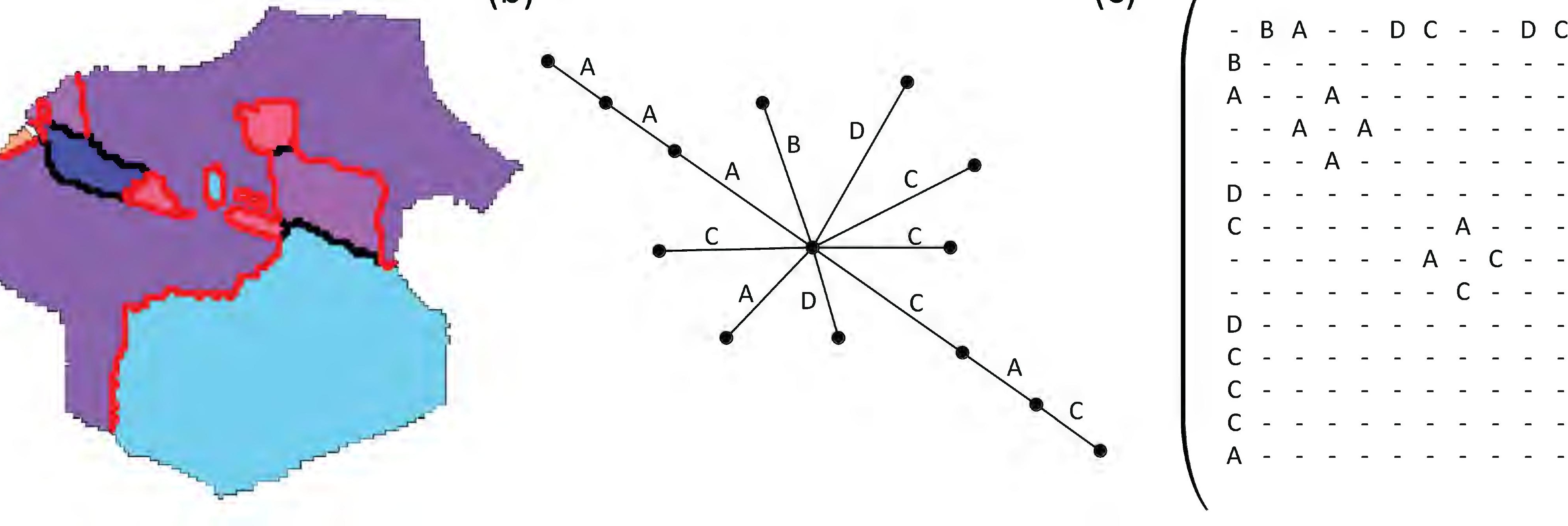

(a)

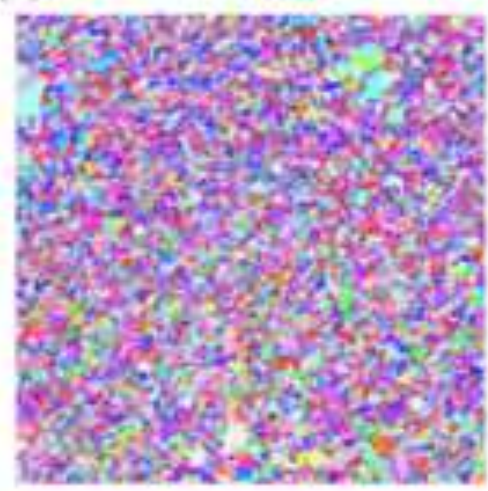

Grain Orientation Spread

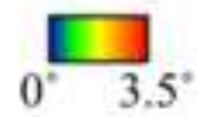

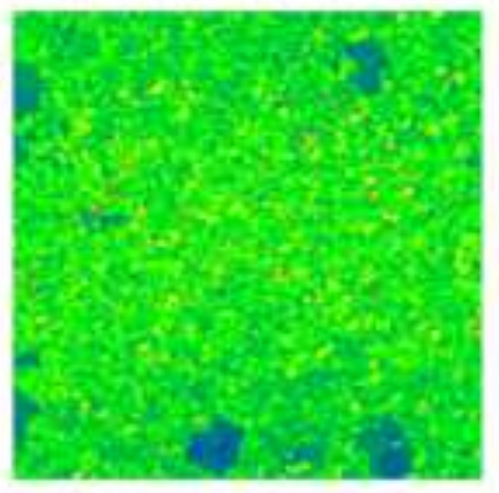

Grain Boundary
Map

Twin Related

Domain map

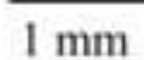

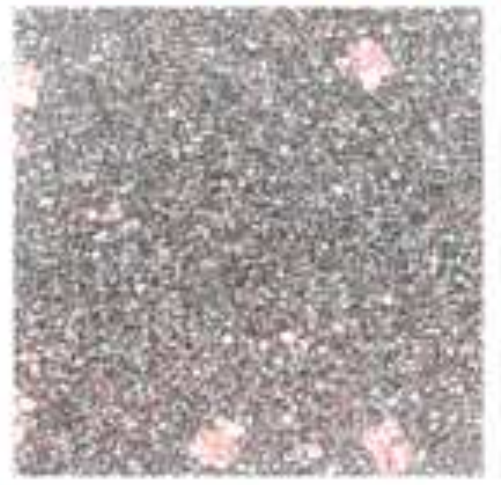

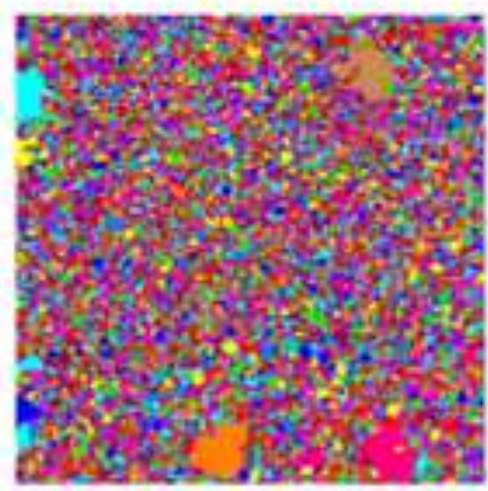

(b)
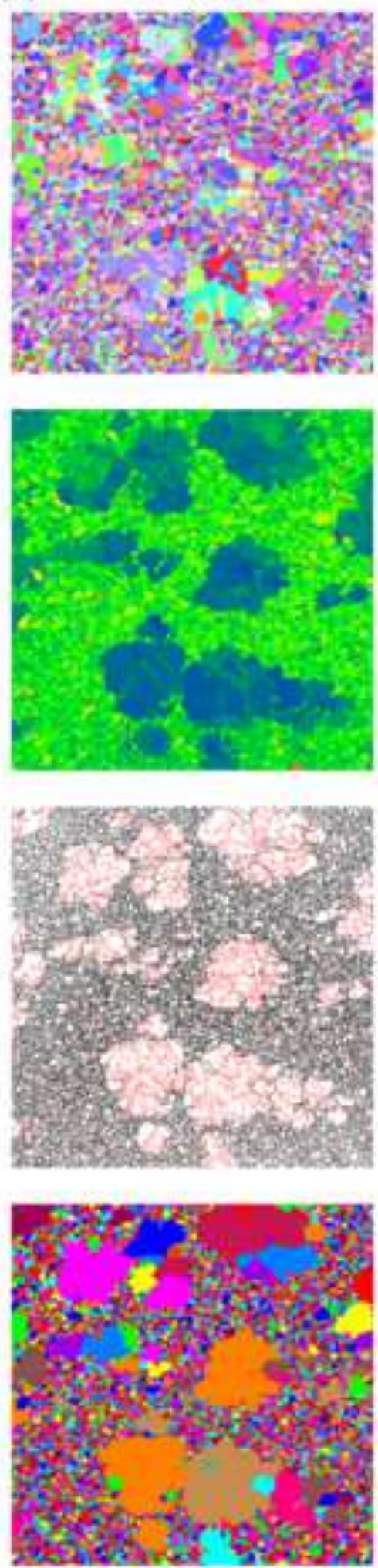

(c) $8.5 \mathrm{~min}$
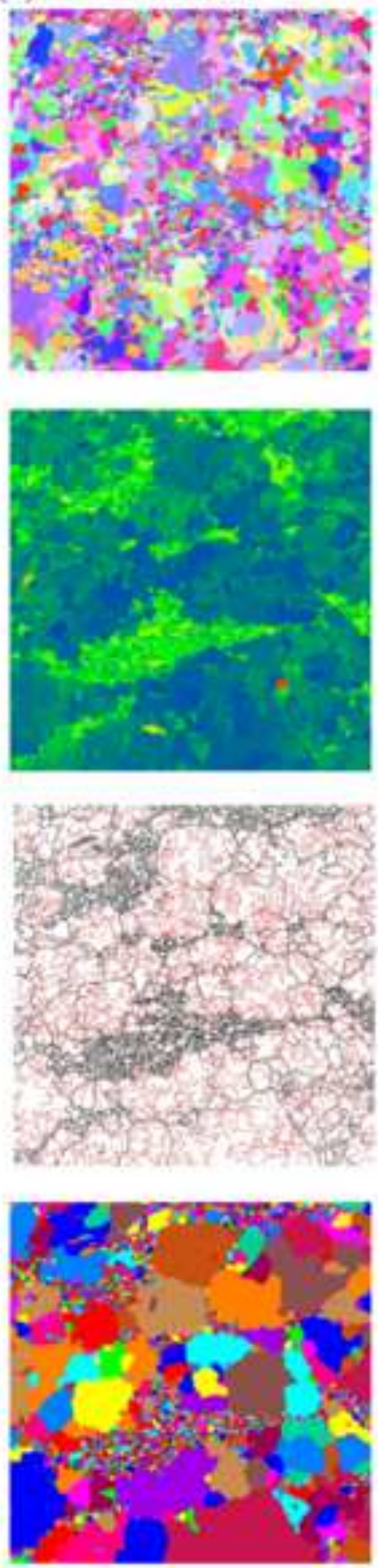

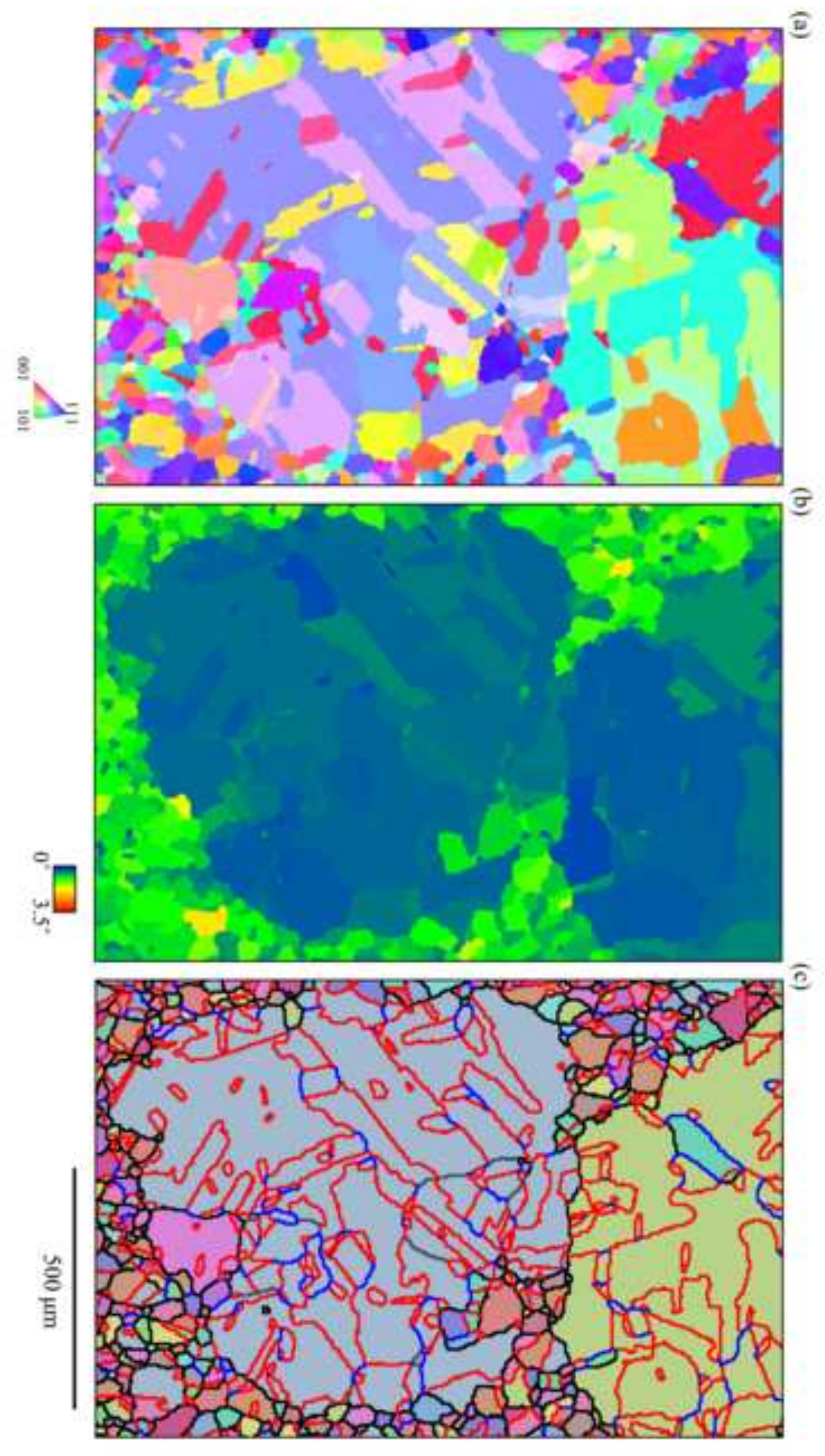

Figure 3

g्

동 


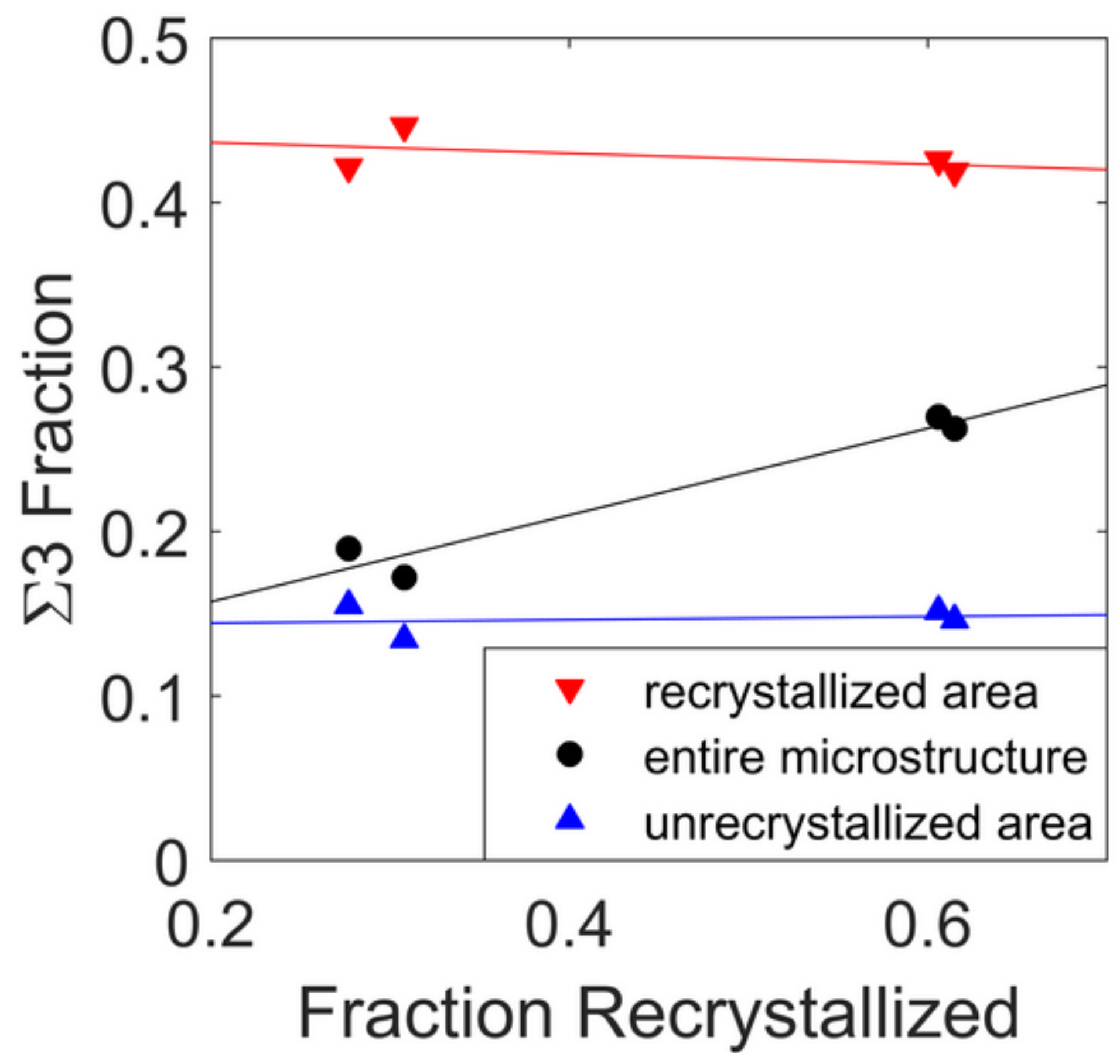




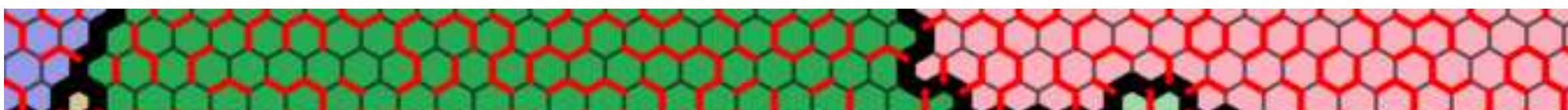
12. QP: MQR

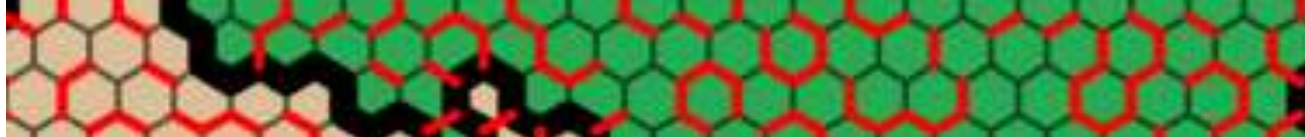

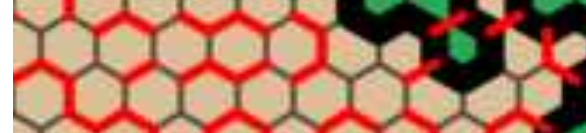
romperate

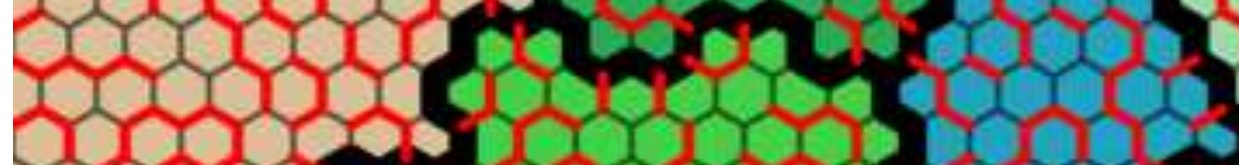

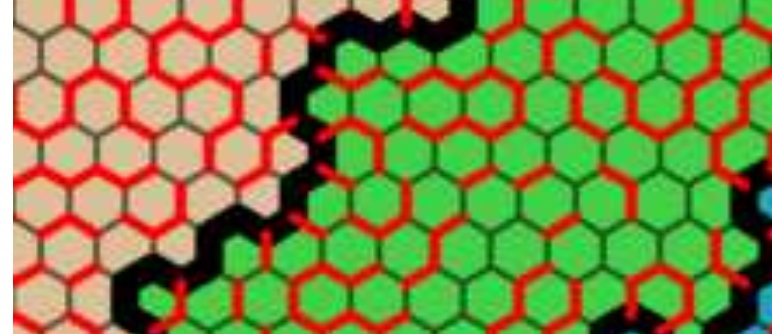

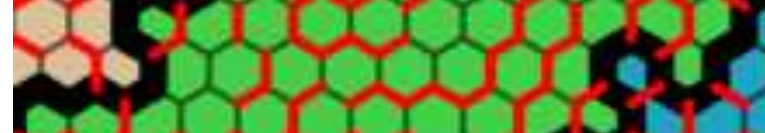

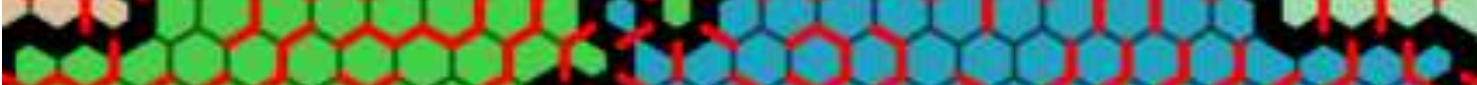

Pen

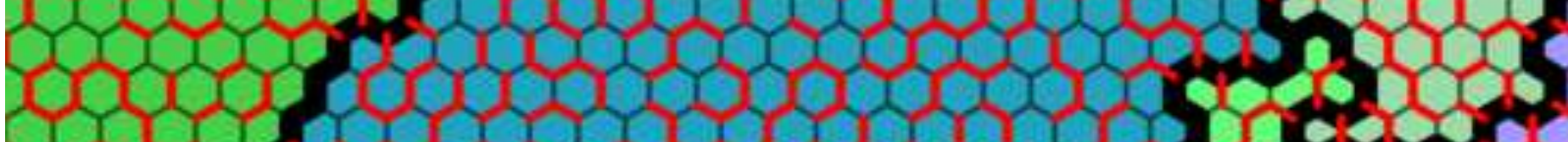

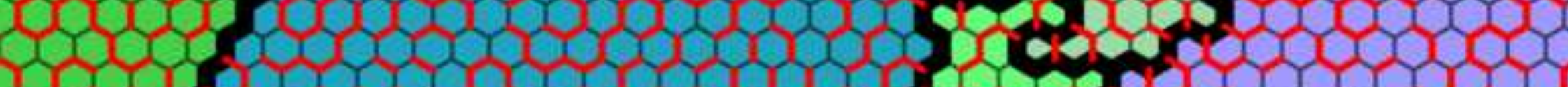
Xop She Sm Losk3 


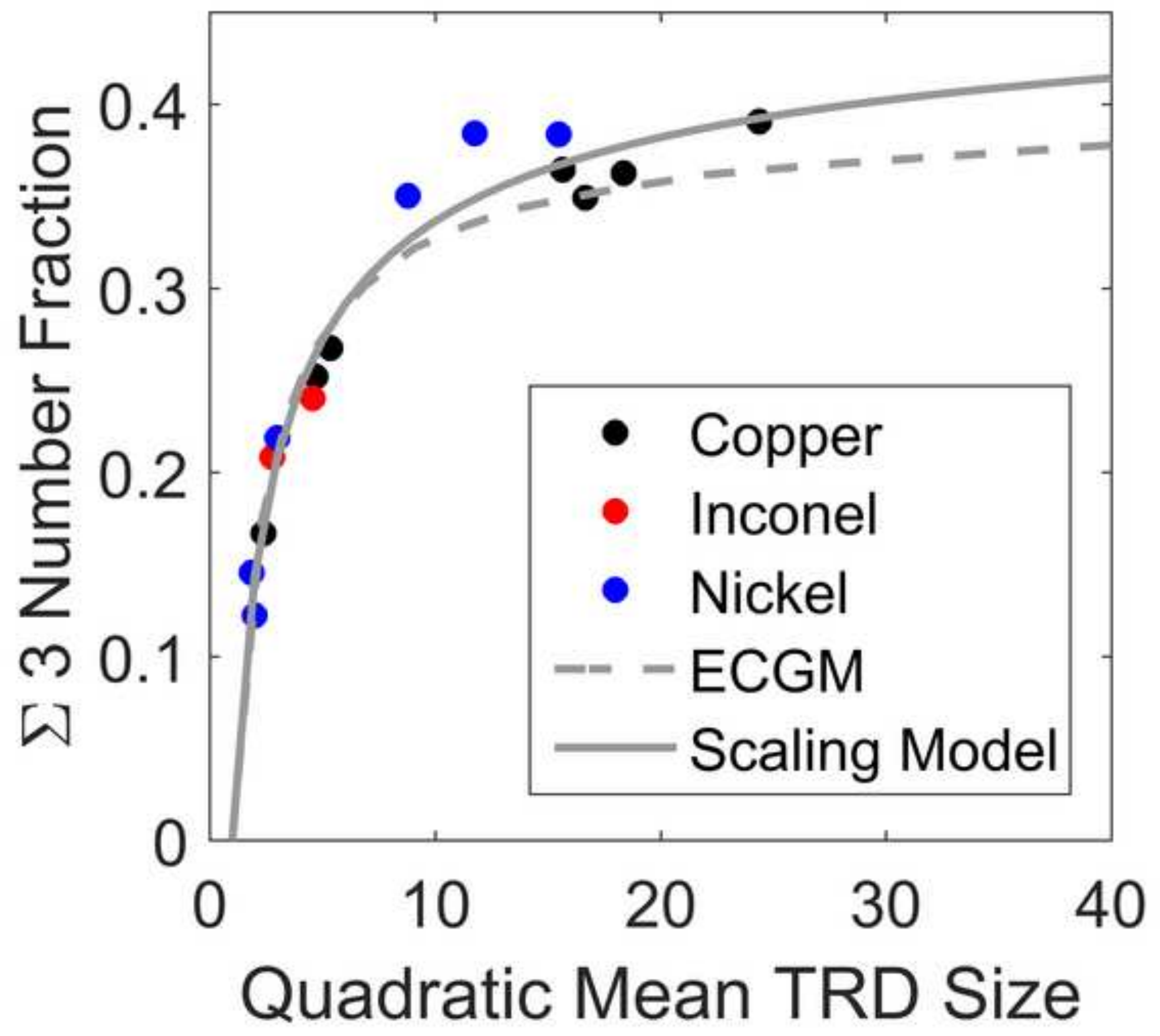


(a)

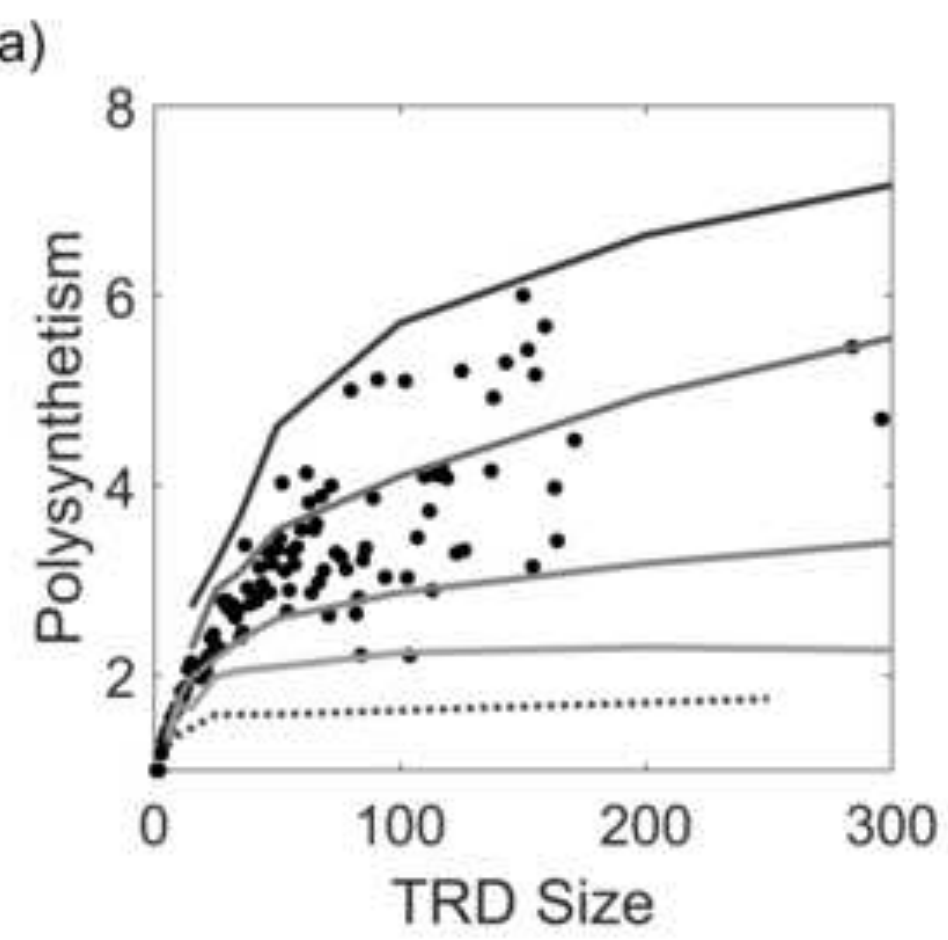

(b)

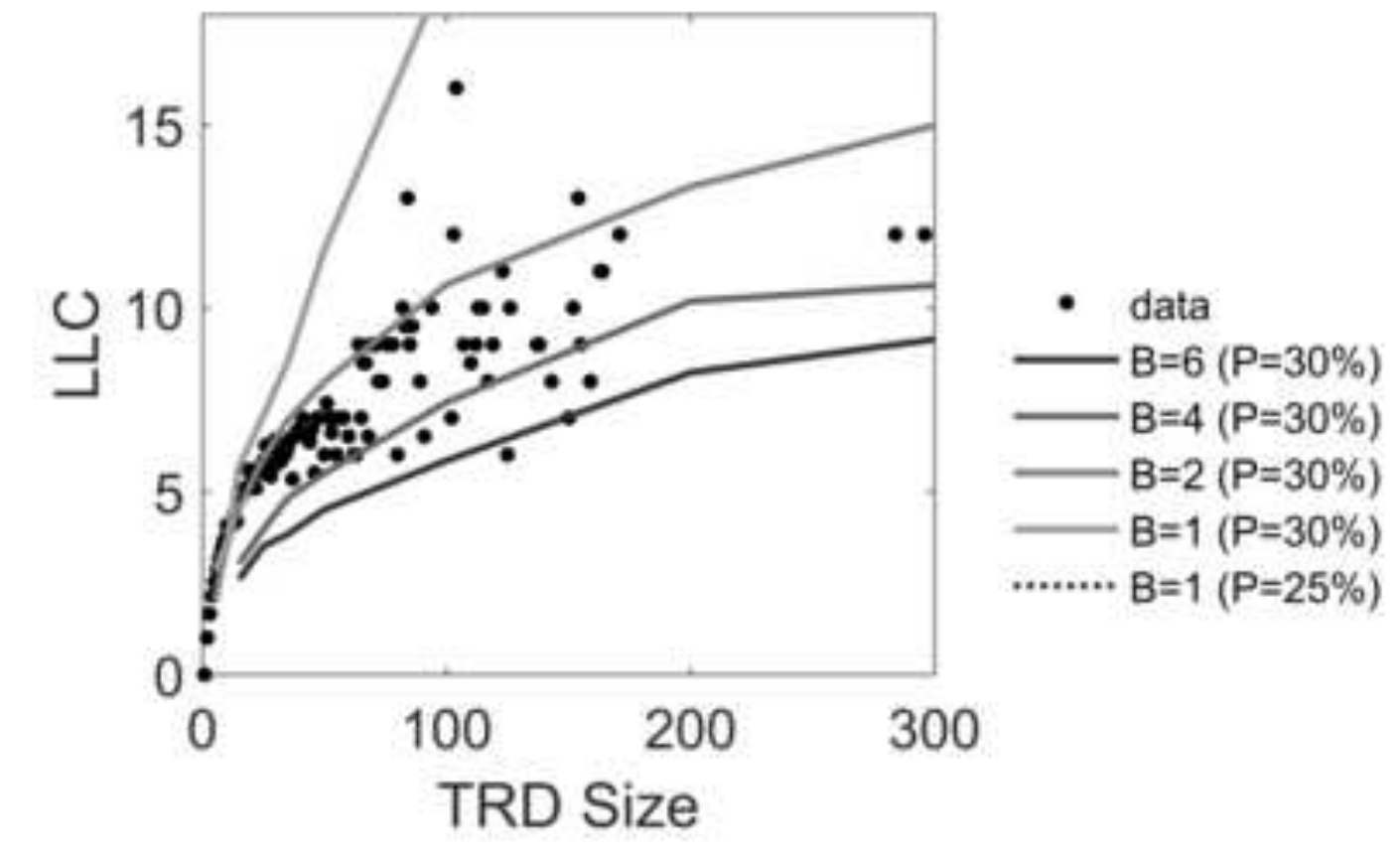



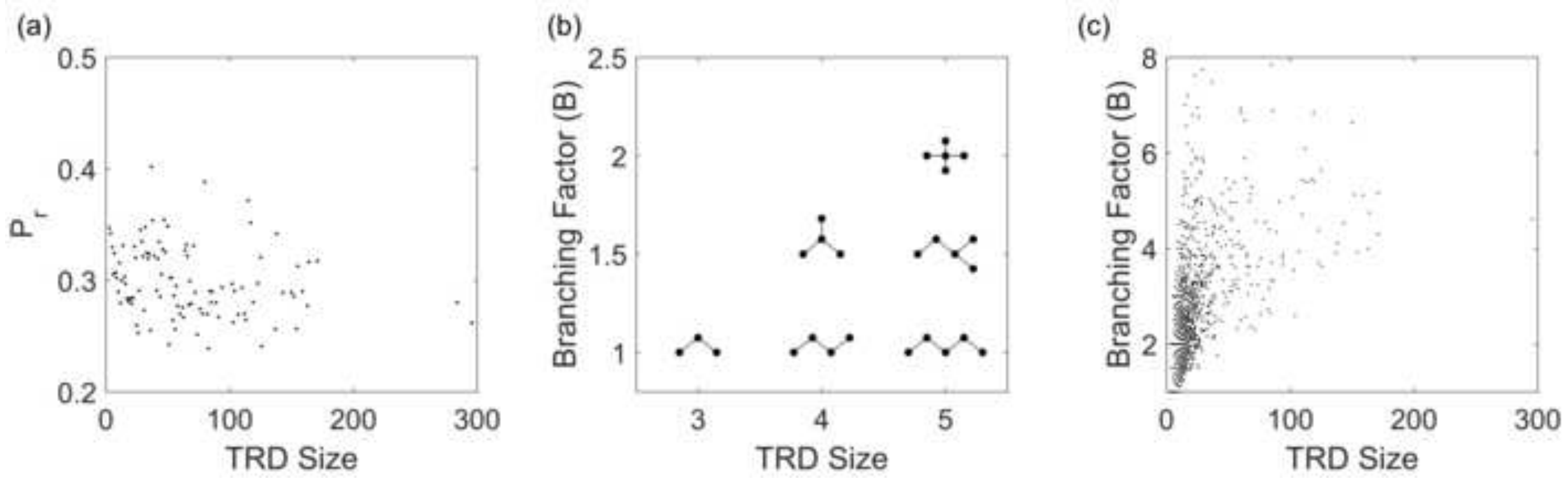
Grain Orientation

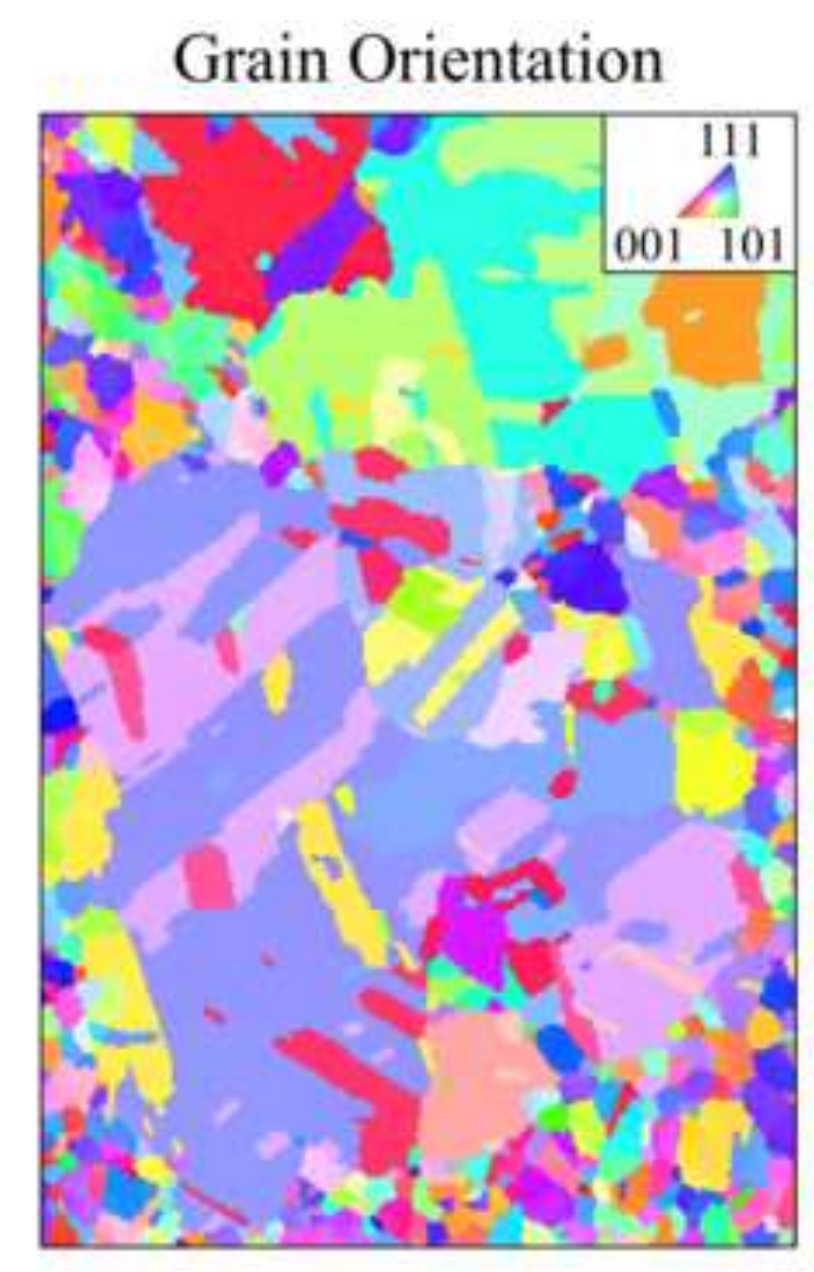

Grain Orientation Spread

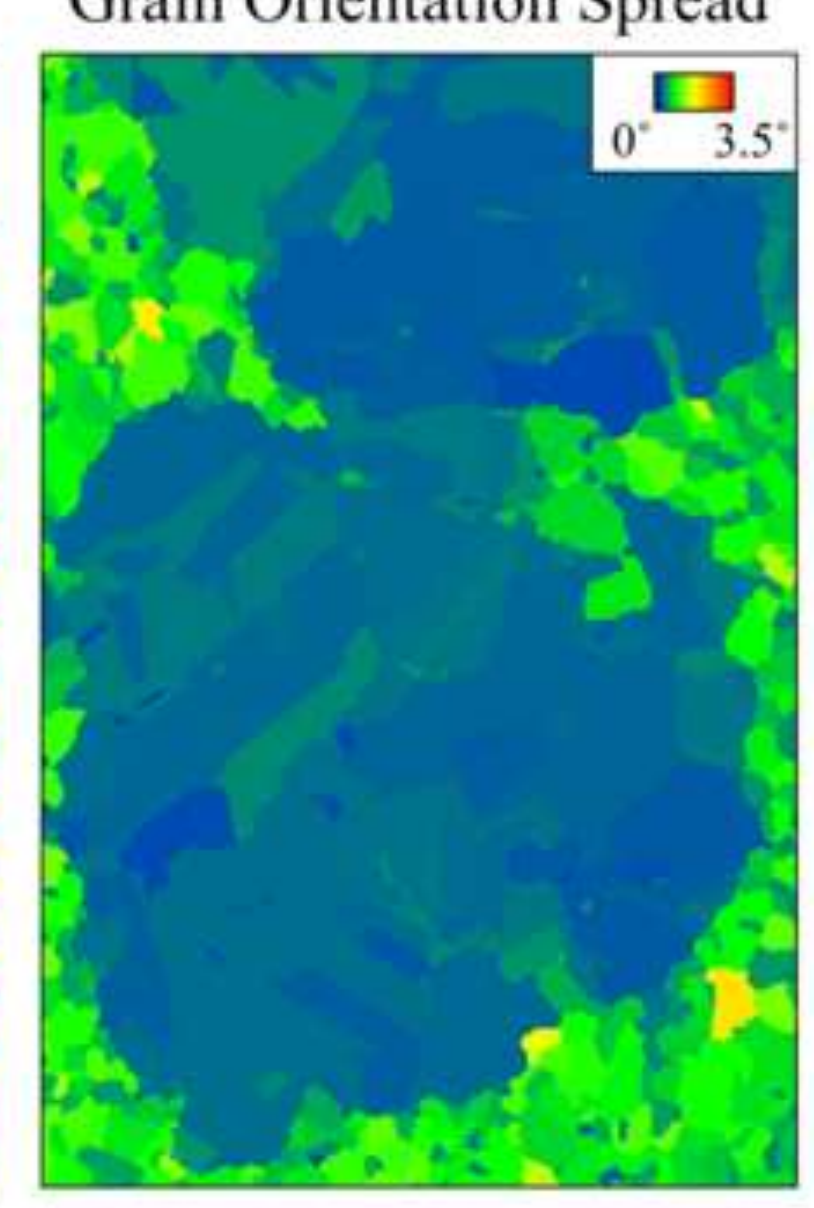

Twin Related Domains

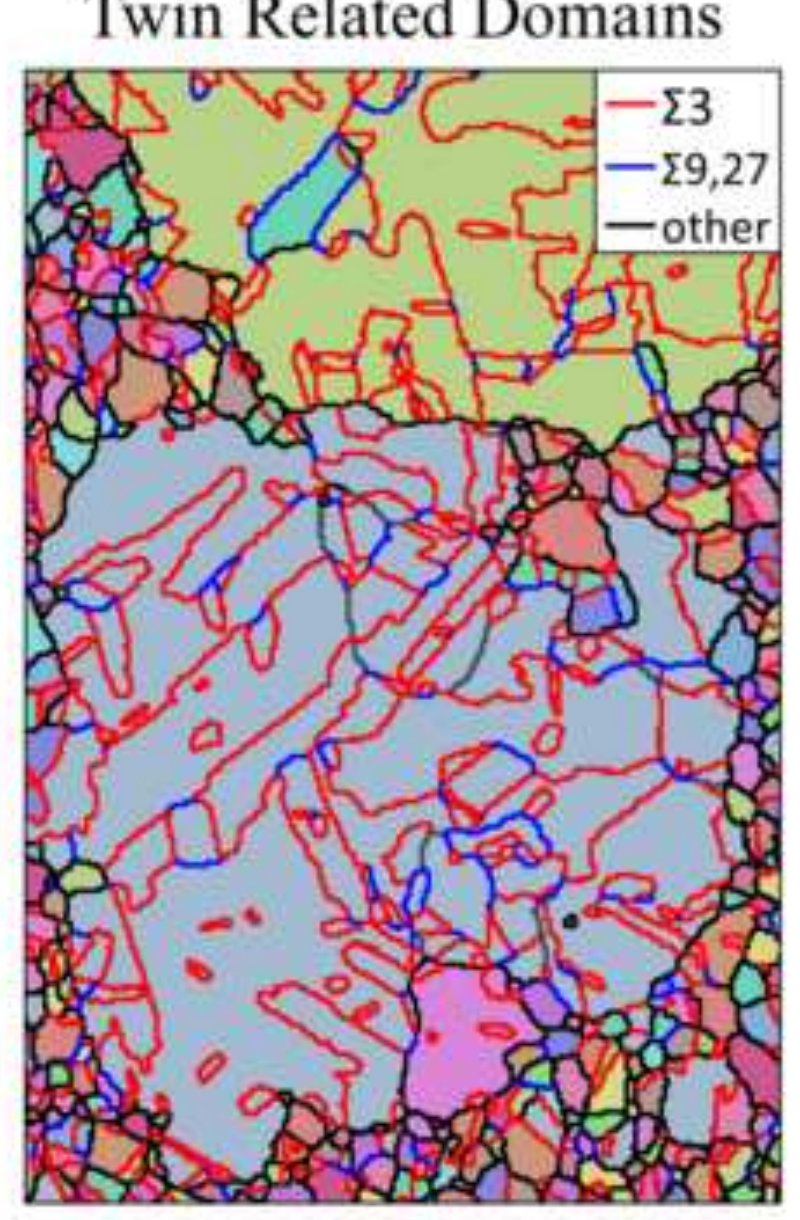

